\title{
Granulocyte colony-stimulating factor attenuates monocrotaline-induced pulmonary hypertension by upregulating endothelial progenitor cells via the nitric oxide system
}

\author{
JUN-FENG LIU ${ }^{1,2}$, ZHONG-DONG DU ${ }^{3}$, ZHI CHEN ${ }^{3}$, ZHONG-CHAO HAN $^{4}$ and ZHI-XU HE ${ }^{1}$ \\ ${ }^{1}$ Laboratory of Tissue Engineering and Stem Cells, Guiyang Medical College, Guiyang, Guizhou 550004; \\ ${ }^{2}$ Department of Pediatrics, The General Hospital of Huabei Oil Field Company, Renqiu, Hebei 062552; \\ ${ }^{3}$ Department of Cardiology, Beijing Children's Hospital, Capital Medical University, Beijing 100045; \\ ${ }^{4}$ National Engineering Research Center of Cell Products, AmCellGene Co. Ltd, Tianjin 300457, P.R. China
}

Received May 27, 2013; Accepted September 24, 2013

DOI: $10.3892 / \mathrm{etm} .2013 .1328$

\begin{abstract}
Granulocyte colony-stimulating factor (G-CSF) has exhibited efficacy at preventing the progression of pulmonary hypertension $(\mathrm{PH})$; however, the exact mechanism is not completely clear. The aim of the present study was to assess whether this protective effect was mediated by the upregulation of circulating endothelial progenitor cells (EPCs) via the nitric oxide (NO) system. PH was induced in male Sprague-Dawley (SD) rats by the administration of a single subcutaneous injection of monocrotaline (MCT). The rats were treated with recombinant human G-CSF (rhG-CSF, $50 \mu \mathrm{g} / \mathrm{kg} /$ day) by subcutaneous injection from day five to day seven subsequent to the injection of MCT. N $\omega$-nitro-L-arginine methyl ester (L-NAME, $4 \mathrm{mg} / \mathrm{kg} /$ day) was intragastrically administered in addition to rhG-CSF as a negative intervention. The changes in hemodynamics and histology, the number and function of circulating EPCs and the concentration of plasma NO were evaluated. With the occurrence of $\mathrm{PH}$ in the rat model, the number and function of circulating EPCs were demonstrated to be markedly downregulated. Moreover, a reduced plasma concentration of NO was observed, which was positively correlated with the number of circulating EPCs. Administration of rhG-CSF elevated the plasma level of NO, upregulated the number and function of circulating EPCs and effectively improved pulmonary hemodynamics and vascular reconstruction. Furthermore, the positive correlation between the levels
\end{abstract}

Correspondence to: Professor Zhi-Xu He, Laboratory of Tissue Engineering and Stem Cells, Guiyang Medical College, 9 Beijing Road, Guiyang, Guizhou 550004, P.R. China

E-mail: zhixu_he@126.com

Key words: pulmonary hypertension, model, endothelial progenitor cells, nitric oxide, granulocyte colony-stimulating factor, hemodynamics of plasma NO and circulating EPCs was also observed in the rhG-CSF treatment group. However, the protective effect of rhG-CSF in PH was attenuated by L-NAME, which mediated the downregulation of NO and the EPCs. Thus, the present study suggests that G-CSF may attenuate the progression of MCT-induced PH by improving vascular injury repair mechanisms via the NO-mediated upregulation of EPCs.

\section{Introduction}

The term pulmonary hypertension $(\mathrm{PH})$ describes a group of lung disorders characterized by a progressive increase in pulmonary arterial pressure. The common pathological feature of PH is pulmonary vascular remodeling. Persistent high blood pressure in the pulmonary circulation results in right ventricular hypertrophy and dilatation, leading to the eventual occurrence of right heart failure. Along with the advancement in studies on $\mathrm{PH}$ in recent years, the importance of endothelial progenitor cells (EPCs) has been recognized among the possible factors involved in the mechanism of PH. EPCs have been considered to be pivotal in maintaining the normal structure and function of the vascular endothelium (1-3), and the dysfunction of this process may, to a certain degree, contribute to the occurrence of pulmonary vascular remodeling in $\mathrm{PH}$. It has been shown that the excessive apoptosis of pulmonary artery endothelial cells in PH results in the destruction of endothelial integrity (4). Furthermore, the endothelial repair effect of EPCs is not able to be implemented effectively, due to the downregulation in EPC number and function $(5,6)$, leading to an imbalance between the lesion and the repair of the vascular endothelium and the further damage of pulmonary arteries.

EPC transplantation has been shown to be effective at preventing the progression of $\mathrm{PH}$ in laboratory rats $(4,7)$. However, this procedure is likely to be limited in clinical practice due to difficulties in obtaining sufficient EPCs from donors during the effective treatment period; therefore, a safe and convenient protocol to improve EPCs in patients with $\mathrm{PH}$ is preferable. As a regulator of granulocytes, granulocyte-colony 
stimulating factor (G-CSF) has been used for decades, and exhibits reliability in the clinic. Furthermore, the administration of G-CSF in cardiovascular disease leads to the repair of the injured vessel and myocardium by the mobilization of bone marrow EPCs and their precursors (8). It has also exhibited efficacy at preventing the progression of PH (9). However, studies of EPC number and function following the administration of G-CSF in PH are lacking, and the mechanism underlying the protective effect of G-CSF on $\mathrm{PH}$ has not been fully elucidated. It has been demonstrated that nitric oxide (NO), as a signaling molecule, is required for the mobilization of bone marrow EPCs (10), while reducing the apoptosis of EPCs (11), and participating in angiogenesis and vasculogenesis $(12,13)$. It has been shown that the cardioprotective effect of G-CSF is also mediated by the NO system $(14,15)$. Therefore, we proposed that G-CSF attenuates $\mathrm{PH}$ by the NO-mediated upregulation of EPCs.

In the present study, we utilized a rat model of $\mathrm{PH}$, created by the subcutaneous injection of monocrotaline (MCT), and treated the rats with recombinant human G-CSF (rhG-CSF). $\mathrm{N} \omega$-nitro-L-arginine methyl ester (L-NAME) was used concurrently as a negative intervention. The therapeutic effect, number and function of circulating EPCs and the concentration of plasma NO were evaluated, in order to enhance the understanding of the protective mechanisms of rhG-CSF in $\mathrm{PH}$.

\section{Materials and methods}

Animals. Male Sprague-Dawley (SD) rats, aged 8 weeks, were purchased from Capital Medical University (Beijing, China) and housed in specific pathogen-free units of the Division of Laboratory Animals at Capital Medical University. Thirty-two rats were randomly divided into four groups: the model [MCT and phosphate-buffered saline (PBS)], rhG-CSF treatment (MCT and rhG-CSF), L-NAME intervention (MCT, rhG-CSF and L-NAME) and control (PBS) groups. Each group contained eight rats. $\mathrm{PH}$ was induced by a single subcutaneous injection of MCT $(60 \mathrm{mg} / \mathrm{kg}$; Sigma, St. Louis, MO, USA) (16), while PBS was administered to the controls. rhG-CSF (50 $\mu \mathrm{g} / \mathrm{kg} /$ day; Xiamen Amoytop Biotech Co., Ltd., Xiamen, China) or PBS was subcutaneously injected from day five to day seven after the injection of MCT. Furthermore, L-NAME (4 mg/kg/day; Sigma) was intragastrically administered at the same time as the rhG-CSF injection and continued to day 21. All animal studies and protocols were approved by the Institutional Animal Care and Use Committee of Capital Medical University.

Examination of hemodynamics. At day 21, the rats were anesthetized by an intraperitoneal injection of pentobarbital $(50 \mathrm{mg} / \mathrm{kg})$. A polyethylene catheter was inserted into the right ventricle (RV) via the right external jugular vein, and another was targeted at the ascending aorta via the right carotid artery. Right ventricular systolic pressure (RVSP) and mean aortic pressure (MAoP) were recorded using a polygraph (Nihon Kohden Corporation, Tokyo, Japan).

Numbers of EPCs in peripheral blood. Peripheral blood was collected from the right external jugular vein into EDTA-containing tubes and $100 \mu \mathrm{l}$ was incubated with $2 \mu \mathrm{l}$ fluorescein isothiocyanate (FITC)-conjugated goat monoclonal antibody against mouse immunoglobulin $\mathrm{G}$, and $5 \mu 1$ mouse monoclonal antibody against rat vascular endothelial growth factor receptor (VEGFR)-2 (Santa Cruz Biotechnology, Inc., Santa Cruz, CA, USA). Following red cell lysis and washing with PBS, the cells were incubated for a further 20 min with $5 \mu$ allophycocyanin (APC)-conjugated mouse monoclonal antibody against rat CD45 (BD Biosciences, Franklin Lakes, NJ, USA) and $5 \mu 1$ phycoerythrin (PE)-conjugated mouse monoclonal antibody against rat CD34 (BD Biosciences). The samples were subsequently centrifuged for $5 \mathrm{~min}$ at $300 \mathrm{x}$ g and then resuspended in $500 \mu \mathrm{l}$ PBS and evaluated using flow cytometry (BD FACSCalibur Flow Cytometer; BD Biosciences, San Jose, CA, USA). Isotype controls were run in parallel and 100,000 events were recorded. Circulating EPCs were defined as cells positive for CD34 and VEGFR-2, but negative for CD45 (17).

Plasma NO measurement. Peripheral blood was centrifuged for $15 \mathrm{~min}$ at $1,200 \mathrm{x} \mathrm{g}$, prior to $50 \mu \mathrm{l}$ plasma being collected into 96-well plates for the measurement of NO. The Nitric Oxide Assay kit (Beyotime Biotechnology, Haimen, China) was based on Greiss reagent. The concentration of NO was determined by spectrophotometry $(490 \mathrm{~nm})$, following the addition of $50 \mu \mathrm{l}$ Greiss reagents I (1\% sulfanilamide in $0.1 \mathrm{~mol} / 1 \mathrm{HCl})$ and II [0.1\% N-(1-naphthyl-ethylenediamine dihydrochloride)].

EPCs cultured in vitro. Mononuclear cells were isolated from peripheral blood using Ficoll density gradient centrifugation (20 min at $400 \mathrm{x} \mathrm{g}$ without brake) and suspended with endothelial cell growth medium-2 (EGM-2; Lonza Group AG, Basel, Switzerland) in 24-well culture plates pre-coated with fibronectin (Sigma), and incubated at $37^{\circ} \mathrm{C}$ in a humidified environment with $5 \%$ carbon dioxide $\left(\mathrm{CO}_{2}\right)$. Unattached cells were removed by extensive washing on day three, and the culture medium was replaced every two days thereafter. Following seven days of culture, cells were incubated with $10 \mu \mathrm{g} / \mathrm{ml}$ DiI-labeled acetylated low-density lipoprotein (acLDL; Molecular Probes ${ }^{\circledR}$, Invitrogen Life Technologies, Carlsbad, CA, USA). Four hours later, the cells were incubated with $10 \mu \mathrm{g} / \mathrm{ml}$ FITC-conjugated lectin from Ulex europeus agglutinin-1 (FITC-UEA-1; Sigma) for $1 \mathrm{~h}$, following fixation with $4 \%$ paraformaldehyde. The cells were subsequently examined using laser scanning confocal microscopy. Differentiating EPCs were identified by double fluorescence staining, as previously described (18).

Function of EPCs in vitro. EPCs were detached using $0.25 \%$ trypsin following seven days of culture. EPC functions, such as proliferation, adhesion and migration, were assessed as described in a previous study (18). With regard to proliferation, the cells were cultured for $24 \mathrm{~h}$ in 96 -well plates at a density of $10^{5}$ cells $/ \mathrm{ml}$ (200 $\mu \mathrm{l}$ per well), prior to $20 \mu \mathrm{l}$ MTT $(5 \mathrm{~g} / \mathrm{l})$ being added for $4 \mathrm{~h}$. Following this, the media was discarded and $100 \mu$ limethylsulfoxide (DMSO) was added for $10 \mathrm{~min}$. The absorbance was measured at a wavelength of $490 \mathrm{~nm}$. For adhesion, EPCs were incubated with EGM-2 in 24-well plates pre-coated with fibronectin $\left(5 \times 10^{4} /\right.$ well $)$ for $30 \mathrm{~min}$. Subsequent to washing three times with PBS, the attached cells 
were counted in a high power field (HPF). To assess the migratory ability of the cells, a modified Boyden chamber $(8-\mu \mathrm{m}$ pore size) was used. EPCs were suspended in $100 \mu$ l EGM-2 without cytokines, plus $0.5 \%$ fetal bovine serum (FBS), in the upper chamber $\left(5 \times 10^{5} / \mathrm{ml}\right)$ and placed in a 24 -well culture plate containing $600 \mu \mathrm{l}$ EGM-2. Following $24 \mathrm{~h}$ of incubation, the lower membrane of the chamber was fixed with $4 \%$ paraformaldehyde. Migrated cells were counted in a HPF, subsequent to staining with $0.1 \%$ crystal violet.

Histological examination. Lung tissues were removed from the rats subsequent to sacrifice by decapitaiton and fixed in $10 \%$ paraformaldehyde for $24 \mathrm{~h}$. Following this, serial paraffin sections $(5-\mu \mathrm{m})$ were stained with hematoxylin and eosin for light microscopy (magnification, x400). The medial wall thickness of the pulmonary arteriole is expressed as: Wall thickness $(\mathrm{WT}, \%)=[($ medial thickness $\times 2) /$ external diameter $] \times 100(9)$.

Statistical analysis. Data are presented as the mean \pm standard deviation, and were statistically analyzed using SPSS statistical software (version 13.0; SPSS, Inc., Chicago, IL, USA). Differences were compared using one-way analysis of variance (ANOVA) tests. Correlations were calculated according to Pearson's correlation. $\mathrm{P}<0.05$ was considered to indicate a statistically significant difference.

\section{Results}

Characteristics of the experimental rats. Approximately 10 days following the co-administration of MCT and PBS, rats exhibited shortness of breath and a reduction in locomotor activity and food intake compared with controls. In addition, the body weight of the model rats was shown to have decreased $(172.3 \pm 19.6$ versus $208.4 \pm 18.5 \mathrm{~g}, \mathrm{P}<0.05)$. The administration of rhG-CSF resulted in a marked improvement in the status of the rats, and there were no differences in body weight between the rhG-CSF treatment group $(203.7 \pm 19.7 \mathrm{~g})$ and the controls $(\mathrm{P}>0.05)$, while only the level of locomotor activity remained slightly decreased. However, under the negative intervention of L-NAME, the body weight of the rats $(170.5 \pm 18.2 \mathrm{~g})$ was markedly decreased compared with that of the rhG-CSF treatment group $(\mathrm{P}<0.05)$.

Changes in hemodynamics and histology. Twenty-one days following the injection of MCT, the RVSP of the rats in the model group was increased compared with that of the controls $(48.13 \pm 2.85$ versus $27.88 \pm 3.04 \mathrm{mmHg}, \mathrm{P}<0.01)$, while the MAoP was decreased from $120.33 \pm 18.25 \mathrm{mmHg}$ in the control group to $97.24 \pm 17.52 \mathrm{mmHg}$ in the model group. This indicated the occurrence of PH. The administration of rhG-CSF led to the RVSP of the rats being decreased significantly compared with that of the model group $(30.38 \pm 2.83$ versus $48.13 \pm 2.85 \mathrm{mmHg}$, $\mathrm{P}<0.01)$. No differences were detected between the rhG-CSF treatment group and the controls $(\mathrm{P}>0.05)$. The MAoP of the rats in the rhG-CSF treatment group $(113.82 \pm 21.73 \mathrm{mmHg})$ was increased compared with that of the model group $(\mathrm{P}<0.05)$, and was restored to the level of the controls ( $\mathrm{P}>0.05$; Fig. 1E and F).

Histological examination indicated that medial hypertrophy of the pulmonary arteriole smooth muscle was evident in the model group (Fig. 1B) and that the WT of the model group was increased compared with that of the controls $(31.74 \pm 3.09$ versus $13.99 \pm 1.14 \%, \mathrm{P}<0.01)$. The administration of rhG-CSF was shown to markedly attenuate the medial hypertrophy of the pulmonary arteriole smooth muscle (Fig. 1C), while the WT of the rhG-CSF treatment group $(17.31 \pm 1.92 \%)$ was notably decreased compared with that of the model group $(\mathrm{P}<0.01$; Fig. $1 \mathrm{G})$.

The protective effects of rhG-CSF on the MCT-induced rat model of $\mathrm{PH}$ were markedly attenuated by the negative intervention of L-NAME. The RVSP of rats in the L-NAME intervention group $(51.19 \pm 2.93 \mathrm{mmHg})$ was increased significantly compared with that of the rhG-CSF treatment group $(\mathrm{P}<0.01)$, while the MAoP was decreased $(96.25 \pm 17.92 \mathrm{mmHg}$, $\mathrm{P}<0.01$; Fig. 1E and F). Histological examination indicated that medial hypertrophy of the pulmonary arteriole smooth muscle was evident, similar to that observed in the model group (Fig. 1D), and the WT (31.97 $\pm 3.22 \%)$ was increased compared with that of the rhG-CSF treatment group $(\mathrm{P}<0.01$; Fig. $1 \mathrm{G})$.

EPC level in peripheral blood. The number of EPCs in the peripheral blood, assessed using flow cytometry, was significantly lower in the model group 21 days subsequent to the injection of MCT compared with the controls $(0.016 \pm 0.007$ versus $0.031 \pm 0.011 \%, \mathrm{P}<0.01)$. The administration of rhG-CSF was shown to notably increase the number of EPCs $(0.042 \pm 0.013 \%)$ compared with the numbers in the model and control groups $(\mathrm{P}<0.01)$. The intervention of L-NAME attenuated the effect of rhG-CSF on the circulating EPCs $(0.015 \pm 0.007 \%, \mathrm{P}<0.01$; Fig. 2A).

Plasma concentration of NO. The plasma concentration of NO was lower in the model group $(19.66 \pm 2.78 \mu \mathrm{mol} / \mathrm{l})$ than in the control group $(54.31 \pm 3.81 \mu \mathrm{mol} / 1, \mathrm{P}<0.01)$. The administration of rhG-CSF was shown to significantly increase the plasma concentration of NO compared with that in the model group (50.85 \pm 2.64 versus $19.66 \pm 2.78 \mu \mathrm{mol} / 1, \mathrm{P}<0.01)$, and no differences in NO level were observed between the rhG-CSF treatment and control groups $(\mathrm{P}>0.05)$. The administration of L-NAME, which is an inhibitor NO synthase, suppressed the upregulation of plasma NO mediated by rhG-CSF (Fig. 2B). Furthermore, the plasma concentration of NO in each group was positively correlated with the number of circulating EPCs $(\mathrm{P}<0.05$; Fig. 2C-E).

EPC growth in vitro. Following seven days of culture, abundant spindle-like cells were observed to be adhered to the bottom of the culture plate (Fig. 3A). Subsequent to binding with FITC-UEA-1, these cells exhibited green fluorescence (Fig. 3B), and red fluorescence was exhibited following phagocytosis of DiI-acLDL (Fig. 3C). The cells positive for the two labels under laser scanning confocal microscopy (observed as yellow fluorescence) were recognized as EPCs undergoing differentiation (Fig. 3D).

EPC function in vitro. The functions of the EPCs were all downregulated in the model group compared with those of the controls. In the test of proliferation ability in vitro, the absorbance (at $490 \mathrm{~nm}$; OD) of the formazan supernatant (dissolved in DMSO) was $0.49 \pm 0.04$ in the model group, which was decreased compared with that of the control 

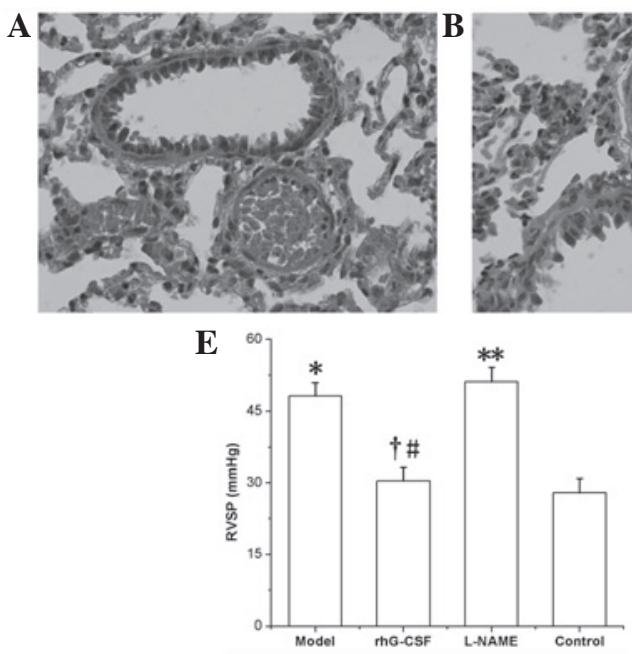
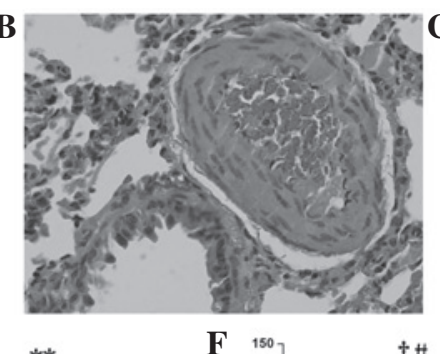
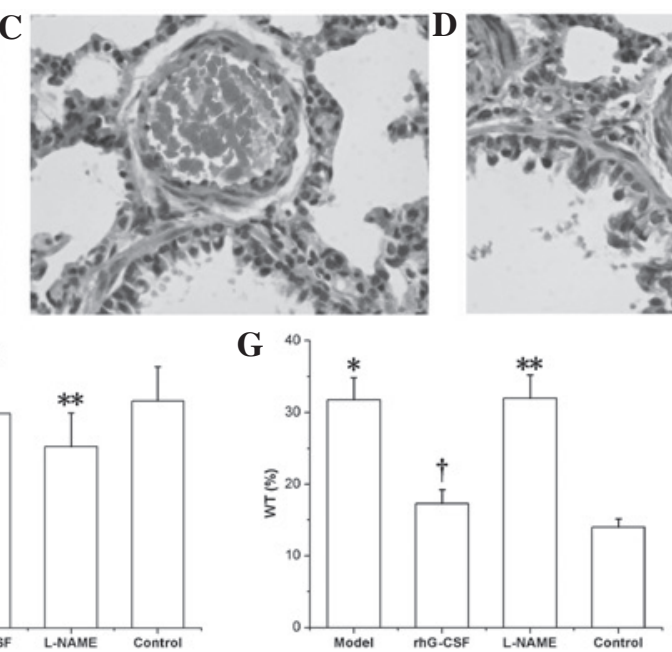

Figure 1. Effect of recombinant human granulocyte colony-stimulating factor (rhG-CSF) on pulmonary hypertension (PH). (A-D) Optical photomicrographs of lung stained with hematoxylin and eosin (magnification, $\mathrm{x} 400$ ). (A) Control group, (B) model group, (C) rhG-CSF treatment group and (D) N $\omega$-nitro-L-arginine methyl ester (L-NAME) intervention group. (E-G) Changes in (E) right ventricular systolic pressure (RVSP), (F) mean aortic pressure (MAoP) and (G) wall thickness (WT) in each group. Data are presented as the mean \pm standard deviation, $n=8$. ${ }^{*} \mathrm{P}<0.01$ compared with the control group; ${ }^{\dagger} \mathrm{P}<0.01$ compared with the model group; ${ }^{*} \mathrm{P}>0.05$ compared with the control group; ${ }^{* *} \mathrm{P}<0.01$ compared with the rhG-CSF treatment group.

A
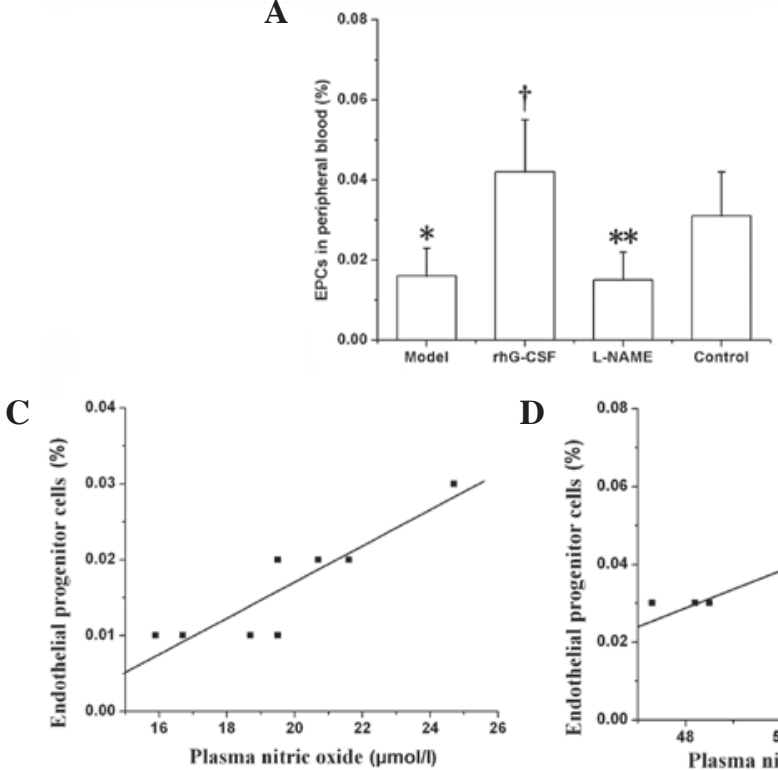

B
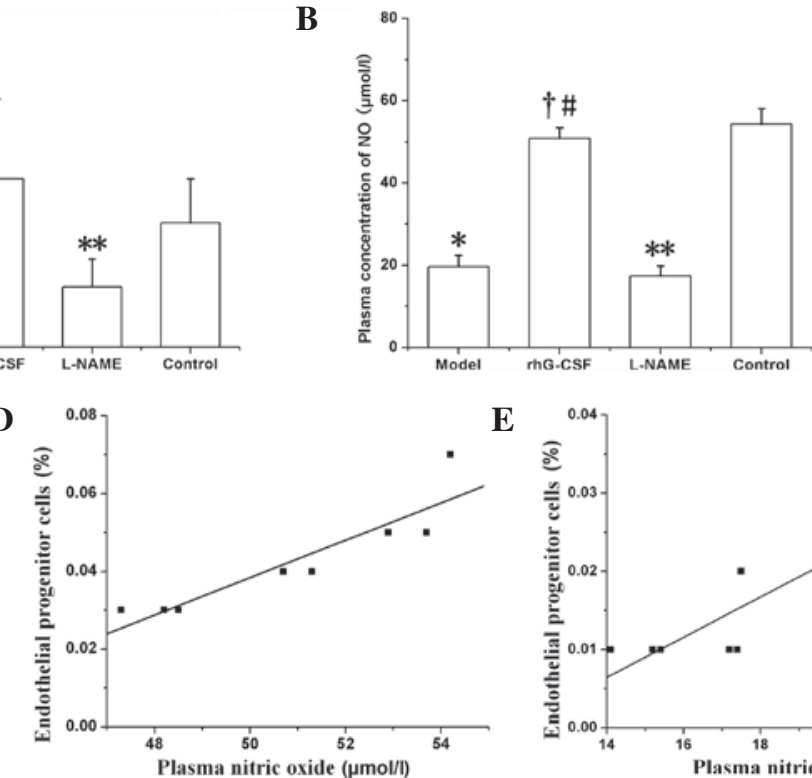

(0.44

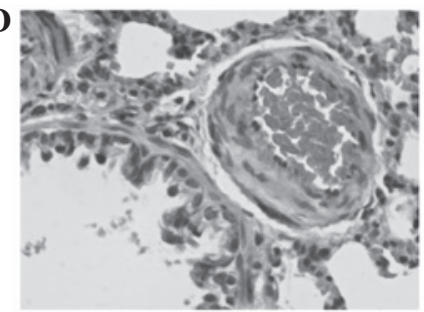

Figure 2. Levels of circulating endothelial progenitor cells (EPCs) and nitric oxide (NO). Data are presented as the mean \pm standard deviation, $\mathrm{n}=8$. (A) Numbers of EPCs in peripheral blood. " $\mathrm{P}<0.01$ compared with the control group; ${ }^{\mathrm{P}} \mathrm{P}<0.01$ compared with the model and control groups; ${ }^{* *} \mathrm{P}<0.01$ compared with the recombinant human granulocyte colony-stimulating factor (rhG-CSF) treatment group. (B) Plasma concentration of NO. "P<0.01 compared with the control group; ${ }^{\mathrm{T}} \mathrm{P}<0.01$ compared with the model group; ${ }^{\mathrm{P}} \mathrm{P}>0.05$ compared with the control group; ${ }^{* *} \mathrm{P}<0.01$ compared with the rhG-CSF treatment group. (C-E) Linear regression correlation between the number of circulating EPCs and the plasma concentration of NO in the (C) model ( $r=0.792$, P $<0.05)$; (D) rhG-CSF treatment $(\mathrm{r}=0.836, \mathrm{P}<0.05)$ and $(\mathrm{E}) \mathrm{N} \omega$-nitro-L-arginine methylester (L-NAME) intervention $(\mathrm{r}=0.889, \mathrm{P}<0.05)$ groups.

group $(0.68 \pm 0.07, \mathrm{P}<0.01)$. Similar results were observed with regard to adhesion ability $(6.93 \pm 1.47$ cells/HPF in the model group versus $11.05 \pm 1.73$ cells/HPF in the control group, $\mathrm{P}<0.01)$ and migratory ability $(7.22 \pm 1.53$ cells/HPF in the model group versus $12.58 \pm 2.15$ cells/HPF in the control group, $\mathrm{P}<0.01)$. In the rhG-CSF treatment group, these functional indices were all upregulated compared with those of the model group (proliferation, OD $0.63 \pm 0.06$; adhesion, $12.35 \pm 1.82$ cells/HPF; migration, $12.97 \pm 2.84$ cells/HPF; $\mathrm{P}<0.01$; Fig. 3F-H). Consistent with the effect of L-NAME on the number of EPCs in the peripheral blood, the functions of the EPCs in the L-NAME intervention group, i.e. proliferation (OD $0.51 \pm 0.04)$, adhesion $(6.73 \pm 1.28$ cells/HPF) and migration (7.18 \pm 1.62 cells/HPF) were all downregulated compared with those of the rhG-CSF treatment group $(\mathrm{P}<0.01$; Fig. 3E-G).

\section{Discussion}

In the present study, it was demonstrated that in a rat model of $\mathrm{PH}$, the number and function of circulating EPCs were mark- 


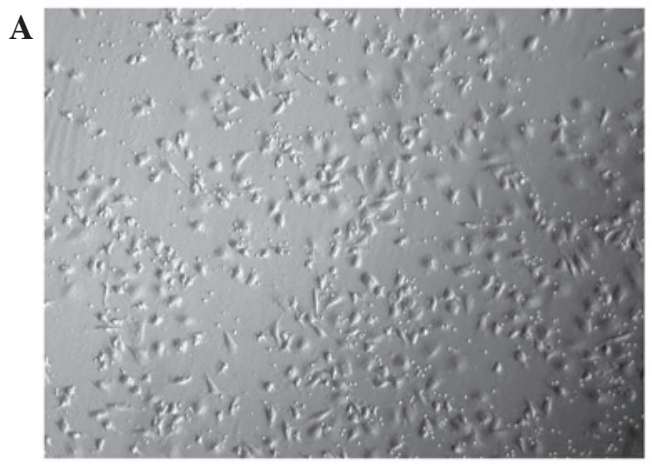

$\mathbf{B}$

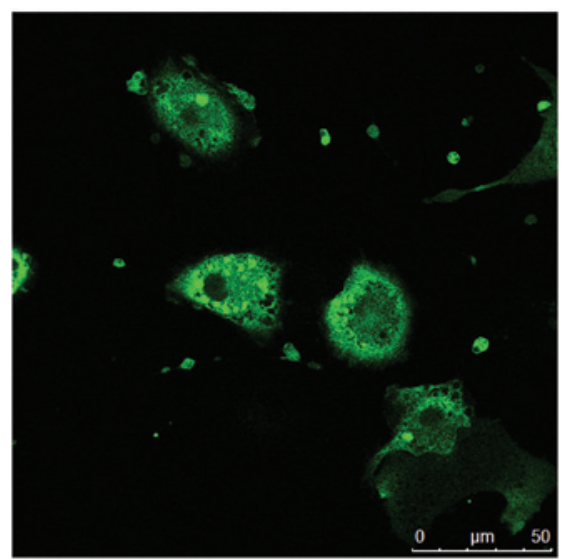

C

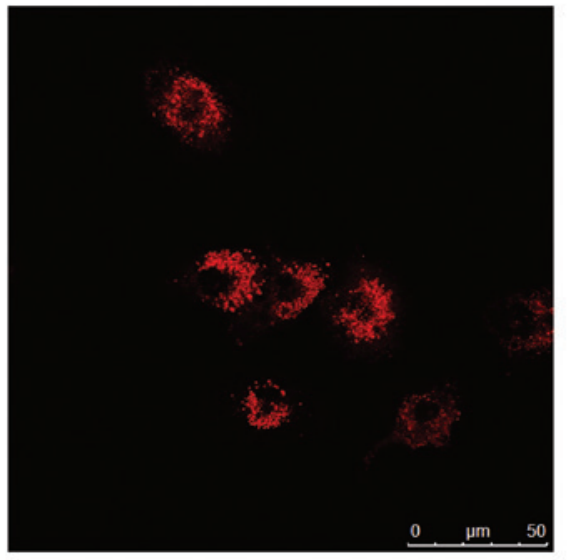

D

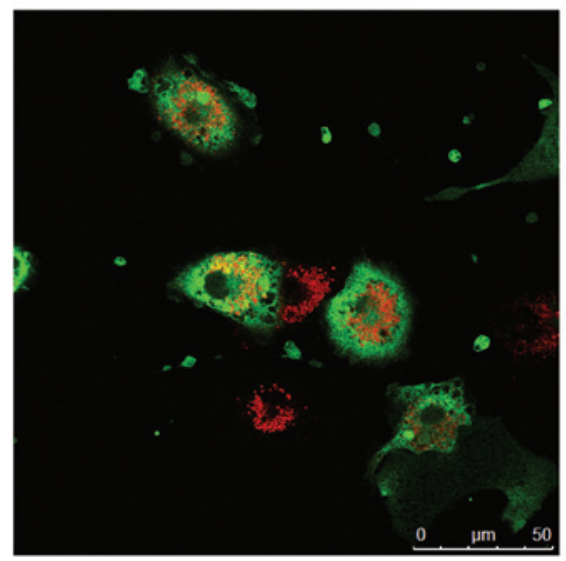

E

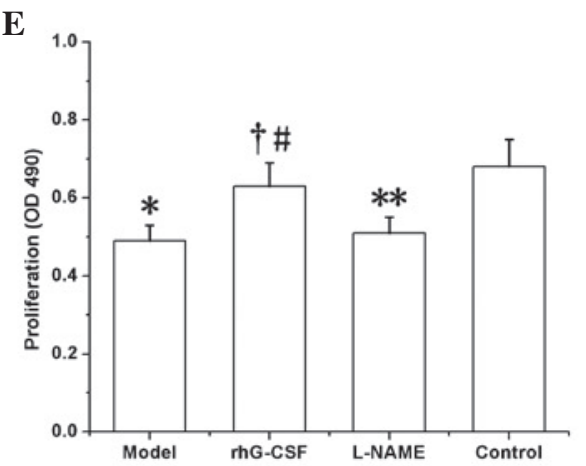

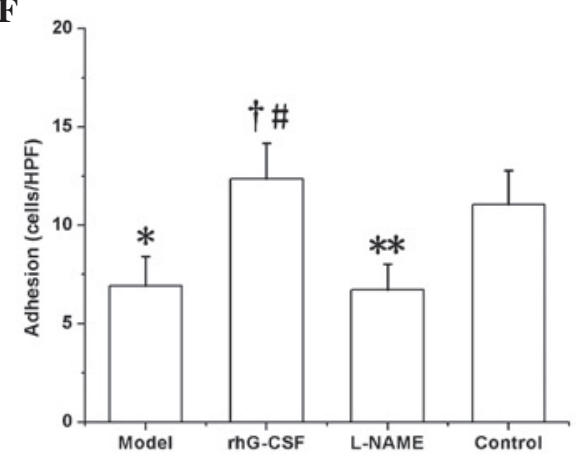

G

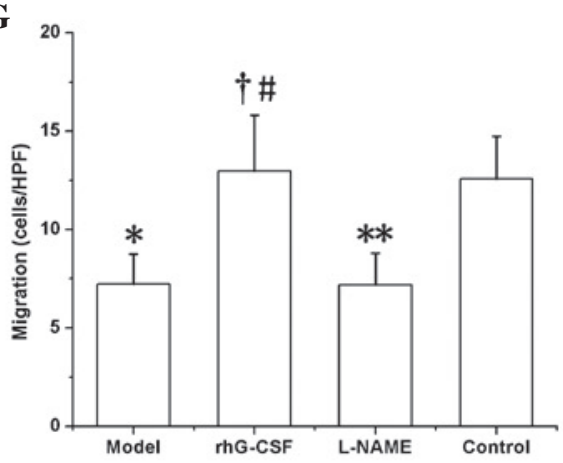

Figure 3. Function of circulating endothelial progenitor cells (EPCs) in vitro. (A-D) Identification of EPCs: EPCs from peripheral blood mononuclear cells of rats were cultured in vitro for seven days. (A) The cells showed a spindle-like morphology, the typical morphology of EPCs (magnification, x100). The majority of the cells were immunopositive for (B) fluorescein isothiocyanate-conjugated lectin from Ulex europeus agglutinin-1 (FITC-UEA-1) and (C) DiI-labeled acetylated low-density lipoprotein (DiI-acLDL). The cells positive for the two labels (see yellow fluorescence) were recognized as EPCs in differentiation (D). (E-G) EPC functions in vitro: (E) Proliferation, (F) adhesion and (G) migration. Data are presented as the mean \pm standard deviation, $n=8$. "P<0.05 compared with the control group; ${ }^{\dagger} \mathrm{P}<0.05$ compared with the model group; ${ }^{*} \mathrm{P}>0.05$ compared with the control group; ${ }^{* *} \mathrm{P}<0.01$ compared with the recombinant human granulocyte colony-stimulating factor (rhG-CSF treatment group).

edly decreased. Moreover, there was a downregulation in the plasma concentration of $\mathrm{NO}$, which was positively correlated with the number of circulating EPCs. Administration of rhG-CSF elevated the plasma level of NO, upregulated the number and function of circulating EPCs and effectively improved pulmonary hemodynamics and vascular reconstruction. Furthermore, the positive correlation between the concentration of plasma NO and circulating EPCs was also observed in the rhG-CSF treatment group. However, the protective effects of rhG-CSF on $\mathrm{PH}$ were impaired by the L-NAME-mediated downregulation of NO and the EPCs.
These results indicate that rhG-CSF prevents or attenuates the progression of MCT-induced PH by improving vascular injury repair mechanisms via the NO-mediated upregulation of EPCs.

$\mathrm{PH}$ is characterized by the persistent contraction and medial hypertrophy of extensive pulmonary arterioles, which may thus result in the abnormal elevation of pulmonary artery pressure. Despite the fact that drug interference may improve the symptoms and decrease the occurrence of heart attack, the effects are partial and limited and right ventricular failure or mortality is likely be the fate of numerous patients in the 
clinic. It is therefore crucial to prevent the progression of the pathological changes in the initial stages of this disease. Studies have shown that the early pathological change in $\mathrm{PH}$ is the injury of the arteriolar endothelium, resulting from excessive apoptosis of the endothelial cells. This then induces vasodilatation dysfunction, the over-proliferation of vascular smooth muscle cells and fibroblasts, ultimately resulting in the occurrence of $\mathrm{PH}$ (19-21). Thus, reconditioning the injured endothelium as early as possible may prevent this course of the disease.

EPCs, which are co-precursors with hematopoietic stem cells, have been suggested to be pivotal to the homeostasis and repair of the vascular endothelium. Studies have shown that the number of circulating EPCs was decreased in patients with $\mathrm{PH}(5,6)$ and that $\mathrm{PH}$ was alleviated by the transplantation of exogenous EPCs in an experimental animal model $(22,23)$, which suggested that the impaired condition of the EPCs contributed to the occurrence of $\mathrm{PH}$. An appropriate strategy to upregulate EPCs may be used to prevent this course effectively. In the present study, the changes in pulmonary hemodynamics and histology that were evident following the injection of MCT in SD rats appeared consistent with those in patients with $\mathrm{PH}$ and were suited to performing further observations on circulating EPCs. It was observed that the number of EPCs in the peripheral blood decreased significantly in the $\mathrm{PH}$ models compared with the number in the controls. In addition, when cultured in vitro, the functions of EPCs, such as proliferation, adhesion and migration, were downregulated. It is possible that the repair processes secondary to the ongoing lesion of the vascular endothelium may lead to the large consumption of circulating EPCs and potentially exhaustion. Bone marrow EPCs and precursors, as the reservoir of circulating EPCs, may also be impaired, in number and function $(24,25)$. A reduction in the protective mechanism of EPCs therefore exacerbates vascular damage and dysfunction.

Data have shown that G-CSF is able to increase the number of circulating EPCs, upregulate the maturation and proliferation capacity of EPCs (26) and then accelerate the repair of the injured vessel and myocardium. Furthermore, G-CSF has exhibited a protective effect in cardiovascular diseases (27). The mobilization of bone marrow stem cells using G-CSF has also been shown to effectively to prevent the progression of $\mathrm{PH}$ (9). In the present study, it was demonstrated that, following the administration of rhG-CSF, the number of EPCs in the peripheral blood was increased significantly and the function of the EPCs was upregulated. As a result, there was an improvement in the pathological changes in the pulmonary artery, in addition to an alleviation of pulmonary artery pressure.

To further investigate the possible mechanism underlying the protective effect of G-CSF in $\mathrm{PH}$, we measured the plasma concentration of NO in each group. It was shown that the level of plasma NO decreased significantly in the rat model of $\mathrm{PH}$ compared with the controls, and that the administration of rhG-CSF effectively induced the upregulation of NO and accelerated the repair of the injured pulmonary artery endothelium by mobilizing the bone marrow EPCs (10), while reducing the apoptosis of EPCs (11). Furthermore, the level of NO was demonstrated to be positively correlated with the number of circulating EPCs in the PH model and rhG-CSF treatment groups. However, the exact signaling pathway involved in the G-CSF-induced upregulation of NO and EPCs was not elucidated in the current study. The study by Ueda et al (14) demonstrated that G-CSF phosphorylated and activated endothelial NO synthase (NOS) in the acute stage of myocardial infarction and increased NO production, thus inducing protective effects on the myocardium. Our observation regarding the change in plasma NO levels in $\mathrm{PH}$ was consistent with that of the study by Ueda et al, which suggests that the same signaling pathway may be involved. Furthermore, the administration of L-NAME, which acted as an NOS inhibitor in the present study, was accompanied by reductions in the plasma concentration of NO and in the number and function of circulating EPCs, thus attenuating the protective effect of rhG-CSF in $\mathrm{PH}$. This further demonstrated the protective effects of the NO-mediated upregulation of EPCs in $\mathrm{PH}$.

In conclusion, the current study indicated that the administration of rhG-CSF may represent a novel strategy for the treatment of $\mathrm{PH}$. The treatment may effectively prevent the disorder by upregulating the number and function of circulating EPCs via the NO system, and then accelerate the reparation of the pulmonary artery endothelial lesion. However, the prospective efficacy and side-effects of rhG-CSF remain important issues to be investigated in advanced experimental and in vivo studies.

\section{Acknowledgements}

This study was supported by the National Natural Science Foundation of China (30560159).

\section{References}

1. Asahara T, Murohara T, Sullivan A, Silver M, van Der Zee R, Li T, Witzenbichler B, Schatteman G and Isner JM: Isolation of putative progenitor endothelial cells for angiogenesis. Science 275: 964-967, 1997

2. Fan Y, Shen F, Frenzel T, Zhu W, Ye J, Liu J, Chen Y, Su H, Young WL and Yang GY: Endothelial progenitor cell transplantation improves long-term stroke outcome in mice. Ann Neurol 67: 488-497, 2010.

3. Bhattacharya V, McSweeney PA, Shi Q, Bruno B, Ishida A, Nash R, Storb RF, Sauvage LR, Hammond WP and Wu MH: Enhanced endothelialization and microvessel formation in polyester grafts seeded with $\mathrm{CD} 34^{+}$bone marrow cells. Blood 95 : 581-585, 2000

4. Zhao YD, Courtman DW, Deng Y, Kuqathasan L, Zhang Q and Stewart DJ: Rescue of monocrotaline-induced pulmonary arterial hypertension using bone marrow-derived endothelial-like progenitor cells: efficacy of combined cell and eNOS gene therapy in established disease. Circ Res 96: 442-450, 2005.

5. Diller GP, van Eijl S, Okonko DO, Howard LS, Ali O, Thum T, Wort SJ, Bédard E, Gibbs JS, Bauersachs J, et al: Circulating endothelial progenitor cells in patients with Eisenmenger syndrome and idiopathic pulmonary arterial hypertension. Circulation 117: 3020-3030, 2008.

6. Junhui Z, Xingxiang W, Guosheng F, Yunpeng S, Furong Z and Junzhu C: Reduced number and activity of circulating endothelial progenitor cells in patients with idiopathic pulmonary arterial hypertension. Respir Med 102: 1073-1079, 2008.

7. Nagaya $N$, Kangawa $K$, Kanda M, Uematsu M, Horio T, Fukuyama N, Hino J, Harada-Shiba M, Okumura H, Tabata Y, et al: Hybrid cell-gene therapy for pulmonary hypertension based on phagocytosing action of endothelial progenitor cells. Circulation 108: 889-895, 2003.

8. Ohki Y, Heissig B, Sato Y, Akiyama H, Zhu Z, Hicklin DJ, Shimada K, Ogawa H, Daida H, Hattori K and Ohsaka A: Granulocyte colony-stimulating factor promotes neovascularization by releasing vascular endothelial growth factor from neutrophils. FASEB J 19: 2005-2007, 2005. 
9. Maruyama H, Watanabe S, Kimura T, Liang J, Nagasawa T, Onodera M, Aonuma K and Yamaguchi I: Granulocyte colony-stimulating factor prevents progression of monocrotaline-induced pulmonary arterial hypertension in rats. Circ J 71: 138-143, 2007.

10. Aicher A, Heeschen C, Mildner-Rihm C, Urbich C, Ihling C, Technau-Ihling K, Zeiher AM and Dimmeler S: Essential role of endothelial nitric oxide synthase for mobilization of stem and progenitor cells. Nat Med 9: 1370-1376, 2003.

11. Laufs U, Werner N, Link A, Endres M, Wassmann S, Jürgens K, Miche E, Böhm M and Nickenig G: Physical training increases endothelial progenitor cells, inhibits neointima formation, and enhances angiogenesis. Circulation 109: 220-226, 2004.

12. Fukumura D, Gohongi T, Kadambi A, Izumi Y, Ang J, Yun CO, Buerk DG, Huang PL and Jain RK: Predominant role of endothelial nitric oxide synthase in vascular endothelial growth factor-induced angiogenesis and vascular permeability. Proc Natl Acad Sci USA 98: 2604-2609, 2001.

13. Guthrie SM, Curtis LM, Mames RN, Simon GG, Grant MB and Scott EW: The nitric oxide pathway modulates hemangioblast activity of adult hematopoietic stem cells. Blood 105: 1916-1922, 2005.

14. Ueda K, Takano H, Hasegawa H, Niitsuma Y, Qin Y, Ohtsuka M and Komuro I: Granulocyte colony stimulating factor directly inhibits myocardial ischemia-reperfusion injury through Akt-endothelial NO synthase pathway. Arterioscler Thromb Vasc Biol 26: e108-113, 2006.

15. Shimada K, Okabe TA, Mikami Y, Hattori M, Fujita M and Kishimoto C: Therapy with granulocyte colony-stimulating factor in the chronic stage, but not in the acute stage, improves experimental autoimmune myocarditis in rats via nitric oxide. J Mol Cell Cardiol 49: 469-481, 2010.

16. Matsuda Y, Hoshikawa Y, Ameshima S, Suzuki S, Okada Y, Tabata T, Sugawara T, Matsumura Y and Kondo T: Effects of peroxisome proliferator-activated receptor gamma ligands on monocrotaline-induced pulmonary hypertension in rats. Nihon Kokyuki Gakkai Zasshi 43: 283-288, 2005 (In Japanese).

17. Chakroborty D, Chowdhury UR, Sarkar C, Baral R, Dasgupta PS and Basu S: Dopamine regulates endothelial progenitor cell mobilization from mouse bone marrow in tumor vascularization. J Clin Invest 118: 1380-1389, 2008.
18. Xu MG, Men LN, Zhao CY, Zhao X, Wang YX, Meng XC, Shen DR, Meng BY, Zhang Q and Wang T: The number and function of circulating endothelial progenitor cells in patients with Kawasaki disease. Eur J Pediatr 169: 289-296, 2010.

19. Gurbanov E and Shiliang X: The key role of apoptosis in the pathogenesis and treatment of pulmonary hypertension. Eur J Cardiothorac Surg 30: 499-507, 2006.

20. Tuder RM, Marecki JC, Richter A, Fijalkowska I and Flores S: Pathology of pulmonary hypertension. Clin Chest Med 28: 23-42, 2007.

21. Budhiraja R, Tuder RM and Hassoun PM: Endothelial dysfunction in pulmonary hypertension. Circulation 109: 159-165, 2004.

22. Ormiston ML, Deng Y, Stewart DJ and Courtman DW: Innate immunity in the therapeutic actions of endothelial progenitor cells in pulmonary hypertension. Am J Respir Cell Mol Biol 43 546-554, 2010.

23. Takahashi M, Nakamura T, Toba T, Kajiwara N, Kato H and Shimizu Y: Transplantation of endothelial progenitor cells into the lung to alleviate pulmonary hypertension in dogs. Tissue Eng 10:771-779, 2004.

24. Valgimigli M, Rigolin GM, Fucili A, Porta MD, Soukhomovskaia O, Malagutti P, Bugli AM, Bragotti LZ, Francolini G, Mauro E, et al: $\mathrm{CD} 34^{+}$and endothelial progenitor cells in patients with various degrees of congestive heart failure. Circulation 110: 1209-1212, 2004.

25. Kissel CK, Lehmann R, Assmus B, Aicher A, Honold J, Fischer-Rasokat U, Heeschen C, Spyridopoulos I, Dimmeler S and Zeiher AM: Selective functional exhaustion of hematopoietic progenitor cells in the bone marrow of patients with postinfarction heart failure. J Am Coll Cardiol 49: 2341-2349, 2007.

26. Powell TM, Paul JD, Hill JM, Thompson M, Benjamin M, Rodrigo M, McCoy JP, Read EJ, Khuu HM, Leitman SF, et al: Granulocyte colony-stimulating factor mobilizes functional endothelial progenitor cells in patients with coronary artery disease. Arterioscler Thromb Vasc Biol 25: 296-301, 2005.

27. Kovacic JC, Muller DW and Graham RM: Actions and therapeutic potential of G-CSF and GM-CSF in cardiovascular disease. J Mol Cell Cardiol 42: 19-33, 2007. 